\title{
Experimental Study of Electric Vehicle Yaw Rate Tracking Control Based on Differential Steering
}

\author{
Cong Li $\mathbb{D}^{1},{ }^{1}$ Yun-Feng Xie, ${ }^{1}$ Gang Wang, ${ }^{2}$ Su-Qi Liu, ${ }^{2}$ Bing Kuang, ${ }^{2}$ and Hui Jing $\mathbb{D}^{2}$ \\ ${ }^{1}$ Guilin University of Aerospace Technology, Guilin 541004, China \\ ${ }^{2}$ Guilin University of Electronic Technology, Guilin 541004, China \\ Correspondence should be addressed to Hui Jing; jinghui@guet.edu.cn
}

Received 12 October 2020; Revised 4 February 2021; Accepted 9 February 2021; Published 22 February 2021

Academic Editor: Kun Wang

Copyright (C) 2021 Cong Li et al. This is an open access article distributed under the Creative Commons Attribution License, which permits unrestricted use, distribution, and reproduction in any medium, provided the original work is properly cited.

This paper investigates the experimental study of differential steering control of a four-wheel independently driven (FWID) electric vehicle (EV) based on the steer-by-wire (SBW) system. As each wheel of FWID vehicle can be independently driven, differential steering is realized by applying different driven torques to the front-two wheels. Firstly, the principle of the differential steering is analyzed based on the SBW system. When the differential steering is activated, the driver's steering request is sent to the vehicle's ECU. Then, the ECU gives different control signals to the front-left and front-right wheels, generating an external steering force on the steering components. The external steering force pushes the steering components to turn corresponding to the driver's request. Secondly, to test the feasibility of differential steering, a FWID EV is assembled and the vehicle is equipped with four independently driven in-wheel motors. The corresponding control system is designed. Finally, the field test of the vehicle based on the proposed differential steering control strategy is performed. In the experiment, the fixed yaw rate tracking and varied yaw rate tracking maneuvers are employed. In the fixed yaw rate tracking, the vehicle can track the desired yaw rate well with differential steering. In addition, the vehicle can track the varied yaw rate with proposed differential steering. The test results confirm the feasibility and effectiveness of the differential steering. By using the differential steering, a backup steering is established without additional components; thus, the costs can be reduced and the reliability of the vehicle steering system can be enhanced, significantly.

\section{Introduction}

With the worldwide concerns on energy conservation and environment protection, electric vehicle has attracted great attention with the advantages of energy reduction and emissions reductions [1-4]. The FWID EV, mounted by in-wheel motors (hub motors), is a promising vehicle architecture for the vehicle maneuverability [5]. On the other hand, to enhance the vehicle handling performance, the SBW system has been equipped on some vehicles. Note that the mechanical connection between the steering wheel and the lower steering assembly in the SBW system is removed; therefore, the reliability of the SBW system becomes more critical, and numerous research studies have been studied on the issue [6-8].

As the FWID EV is driven independently by the in-wheel motors, the driven torque of the front-two in-wheel motors can be different to generate a novel steering mechanism: differential steering. Some similar research studies have been published on the issue, such as differential assisted steering and skid steering [9-11]. The study in [12] investigated the feasibility of differential drive torque assisted steering and the capability of road feel keeping of the control system. In addition, Wang et al. conducted a study on the assistance quality analysis and robust control of electric vehicle with differential drive assisted steering system in [13]. The study in [14] investigated the differential power-assisted steering system of in-wheel electric vehicle based on a collaborative optimization model. The study in [15] proposed an experimentally validated dynamic model to describe the motion of a skid-steered vehicle in the pivotal steering condition, where the experiments were based on a specific $6 \times 6$ unmanned skid-steered vehicle, which was driven by six 
independent in-wheel motors. The study in [16] presented an effective methodology for generating dynamically feasible and energy-efficient trajectories for skid-steered autonomous ground vehicles (AGVs). Reference [17] studied the robust $H_{\infty}$ Takagi-Sugeno fuzzy output-feedback control for differential speed steering vehicles. The differential drive-based yaw stabilization using MPC for distributed-drive articulated heavy vehicle is presented in [18]. What is more, the integrated hierarchical control strategy of active suspension and differential assisted steering system for electric-wheel vehicle is discussed in [19]. The study in [20] presented the motion tracking control for unmanned ground vehicle, consisting of straight-line driving control and cornering control.

The above steering literatures investigated vehicle steering control based on the speed difference between the front-two wheels; and the above differential assisted steering mainly focused on the assisted torque on driver's hands and road feel keeping ability, where no active steering was involved. However, in the SBW system, it is critical to ensure the reliability of the steering system, as well as reducing the additional costs. In addition, compared with the theoretical study of the differential steering, the experimental study of the differential steering is really rare.

To handle the above problems, in this paper, the differential steering is adopted as a backup steering mechanism to enhance the reliability and reduces the external costs in case of the SBW system failure. The main contributions of the paper are as follows. First, the principle of the differential steering is studied based on the analysis of the steering system. Then the FWID EV is assembled and the control system is designed. Finally, the field test of the EV based on the proposed differential steering control strategy is performed and the results are analyzed. The rest of the paper is organized as follows. Section 2 studies the mechanism of the proposed differential steering. Section 3 discusses the control schema of the proposed differential steering and the FWID $\mathrm{EV}$. Section 4 gives the field test of the EV with the yaw rate tracking experiments, followed by the conclusion in Section 5.

\section{Vehicle Differential Steering Dynamics}

As in Figure 1, the principle of differential steering system based on the FWID EV with SBW system is presented, where there is no mechanical connection between steering wheel and steering actuator. The driver steers the steering wheel and the steering request signal is sent to the vehicle's ECU. The steering request can be realized by the electric power steering (EPS). However, when the steering motor completely fails or the steering signal transmission error occurs, the driver's steering request will not be able to be functioned. Meanwhile, the fault diagnosis system detects the steering fault and activates the differential steering to perform the driver's driving request. Note that the fault diagnosis system of SBW system and electric vehicle has been extensively studied previously and this is not the focus of this paper.

As seen in Figure 1, when the regular SBW system is in complete failure and the differential steering is activated to

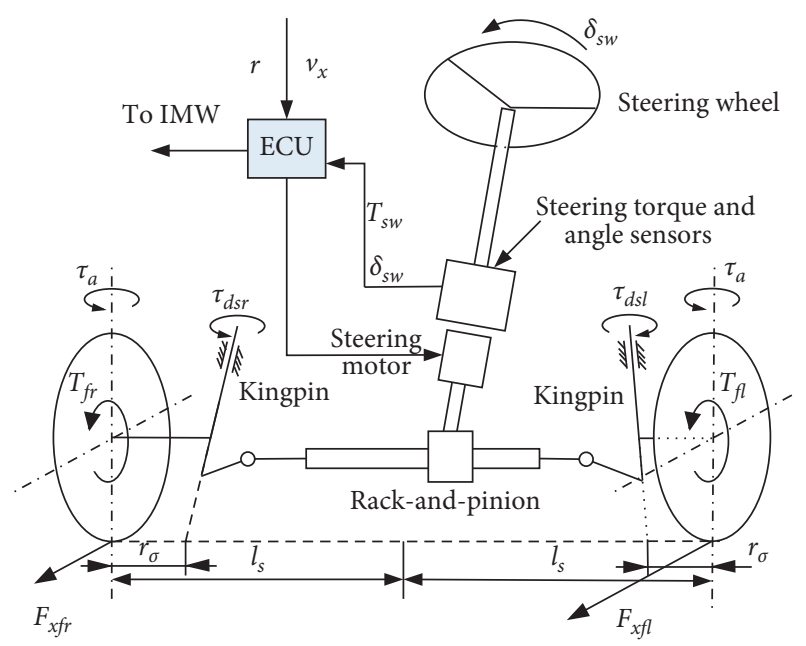

Figure 1: System model with differential steering.

steer the vehicle, the different driven forces of front-two inwheel motors will yield a moment

$$
M_{z^{\prime}}=\tau_{d s 2}-\tau_{d s 1}=\left(F_{x f r}-F_{x f l}\right) r_{\sigma},
$$

where $r_{\sigma}$ denotes the scrub radius, $\tau_{d s i}(i=1,2)$ denotes the moment around kingpin of the $i$ th wheel, and $F_{x f i}(i=r, l)$ denotes the longitudinal tire force of the $i$ th wheel, respectively. Then, the moment will generate a steering angle, which is the focus of the differential steering. In the figure, it is clear that the steering system will turn right when the driven torque of the left wheel is larger than that of the right. The dynamics of the differential steering system is given as follows $[9,10]$ :

$$
J_{e} \ddot{\delta}+b_{e} \dot{\delta}=\tau_{a}+M_{z^{\prime}}
$$

where $J_{e}$ and $b_{e}$ indicate the effective inertia and damping of the steering system, respectively. $\delta$ is the front wheels' steering angle. $\tau_{a}$ denotes the tire self-aligning moment.

According to the brush model [21], the self-aligning moment $\tau_{a}$ can be expressed as $\tau_{a}=\kappa_{1} C_{f} \alpha_{f}$, where $\kappa_{1}$ is a constant, and $C_{f}$ is front tire cornering stiffness. $\alpha_{f}$ is the front wheel slip angle, which can be expressed as $\alpha_{f}=\delta-\left(v_{y} / v_{x}\right)-\left(l_{f} / v_{x}\right) r$, where $v_{x}$ and $v_{y}$ denote the vehicle's longitudinal and lateral velocities, respectively. $l_{f}$ and $l_{r}$ denote the distances from the front axle and the rear axle to the central gravity (CG), respectively.

Assuming that the front wheel steering angle is small, the moment around the center of gravity of the vehicle $M_{z}$ is defined as

$$
M_{z}=\left(F_{x f r}-F_{x f l}\right) l_{s}
$$

where $l_{s}$ denotes the half of the vehicle track width. Then, the moment $M_{z}^{\prime}$ can be deduced as $M_{z}^{\prime}=\kappa_{2} \Delta M_{z}$, with $\kappa_{2}=r_{\sigma} / l_{s}$.

Then, system (2) can be rewritten as

$$
\dot{\delta}=\frac{\kappa_{1} C_{f}}{b_{e}} \delta-\frac{\kappa_{1} C_{f} l_{f}}{b_{e} v_{x}} r+\frac{\kappa_{2}}{b_{e}} M_{z}+d_{1},
$$


where $d_{1}=-\left(\kappa_{1} C_{f} / b_{e} v_{x}\right) v_{y}-\left(J_{e} \ddot{\delta}\right) / b_{e}$ denotes small distances termed as $v_{y}$ and $\ddot{\delta}$ are small normally.

\section{The Vehicle Configuration and the Control Schema}

To verify the differential steering, a FWID EV is refitted from an All Terrain Vehicle (ATV), which has more convenience in equipping the related components. The vehicle configuration is given in Figure 2. The FWID EV is equipped with four in-wheel motors; each of the front-two motors has $6 \mathrm{Kw}$ power, and each of the rear ones has $8 \mathrm{Kw}$ power. The inwheel motor is controlled by the Kelly Motor Controller, where control signal is an analog voltage signal within $[0,5]$ $\mathrm{V}$, and the motor controller works in Torque Mode. It is clear that the Li-ion battery and the MicroAutoBox controller are in the trunk, the dual-antenna GPS is on the roof, and the IMU is near the CG point of the vehicle. The vehicle parameters are listed in Table 1.

The GPS/IMU configuration is given in Figure 3. Note that the GPS Real Time Kinematic (RTK) technology is used to improve the positioning accuracy. The signals are received by the GPS base station from the satellites and then transmitted to the vehicle by a high-power transmitter. The vehicle speed, acceleration, heading angle, and yaw rate signal are measured by the IMU component near the CG of the vehicle.

This paper studies the vehicle handling control of a FWID EV based on the differential steering. The yaw rate $r$, which will influence the vehicle handling performance, should be well regulated in the control system. Then control object is to design a controller, with which the yaw rate tracking error can be minimized. The flow chart of the control schema is shown in Figure 4. In the figure, it is clear that the controller receives two signals: the desired yaw rate $r_{d}$ and the actual yaw rate $r_{m}$. The desired yaw rate signal $r_{d}$ is generated from the driver input, which is an analog voltage signal. For safety reasons, the signal is converted into $[-10$, 10], indicating that the desired yaw rate can be modified from $-10 \mathrm{deg} / \mathrm{s}$ to $10 \mathrm{deg} / \mathrm{s}$. However, the range can be enlarged in the severe driving conditions. The actual yaw rate $r_{m}$ is measured by the IMU near the CG point of the vehicle. Then the yaw rate difference $\Delta d$ between $r_{d}$ and $r_{m}$ is minimized with a PID controller.

As seen in Figure 5, a voltage signal $d V$ is obtained from the PID controller corresponding to yaw rate difference $\Delta d$. Then the voltage $d V$ is converted into two parts, the control voltages $V_{1}$ and $V_{2}$, to control the front-two in-wheel motors, respectively. Considering the dead zone and property of the in-wheel motor controller, an offset is added to keep the motor running in normal conditions.

In Figure 4, the motor controller receives the control signals $V_{1}$ and $V_{2}$ and gives driving commends to the frontleft and front-right in-wheel motors; then the desired torques $T_{1}$ and $T_{2}$ are generated, respectively. Since the steering wheel is released by the driver, the different driven torque between the front-two in-wheel motors will force the fronttwo wheels to rotate around their Kingpin, as well as the steering wheel. Then the vehicle completes the steering

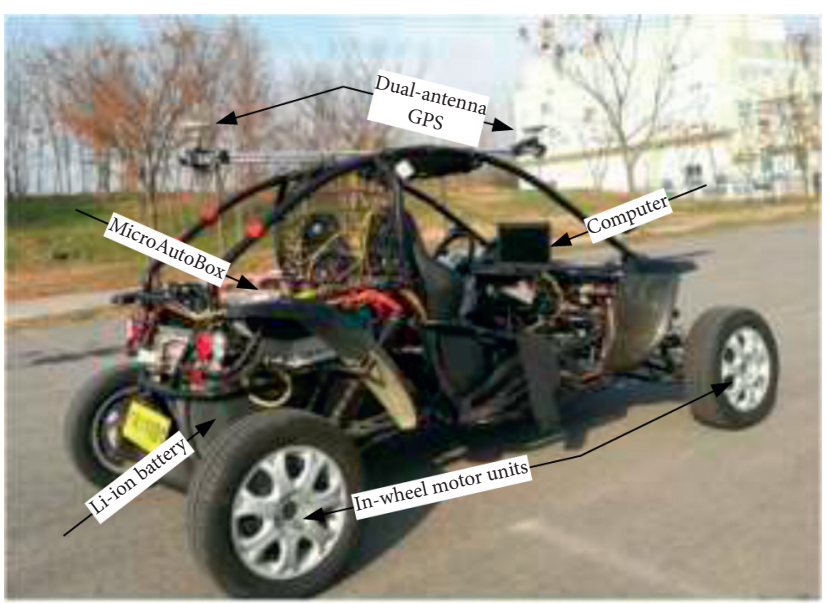

Figure 2: The FWID EV configuration.

TABLE 1: Vehicle parameters.

\begin{tabular}{|c|c|c|}
\hline Symbol & Value & Indicators \\
\hline \multirow{2}{*}{$m$} & $649 \mathrm{~kg}$ & Vehicle mass (without passengers) \\
\hline & $801 \mathrm{~kg}$ & Vehicle mass (with two passengers) \\
\hline$l_{f}$ & $1.2 \mathrm{~m}$ & Distance from the CG to the front axle \\
\hline$l_{r}$ & $1.2 \mathrm{~m}$ & Distance from the CG to the rear axle \\
\hline$l_{s}$ & $0.72 \mathrm{~m}$ & Half length between the front-two wheels \\
\hline $\begin{array}{l}\text { Max. } \\
\text { voltage }\end{array}$ & $72 \mathrm{~V}$ & Vehicle battery voltage \\
\hline Max. power & $28 \mathrm{~kW}$ & Total power of the four motors \\
\hline$V_{1}, V_{2}$ & {$[0,5] \mathrm{V}$} & $\begin{array}{l}\text { Control voltage of the in-wheel motor } \\
\text { controller }\end{array}$ \\
\hline
\end{tabular}

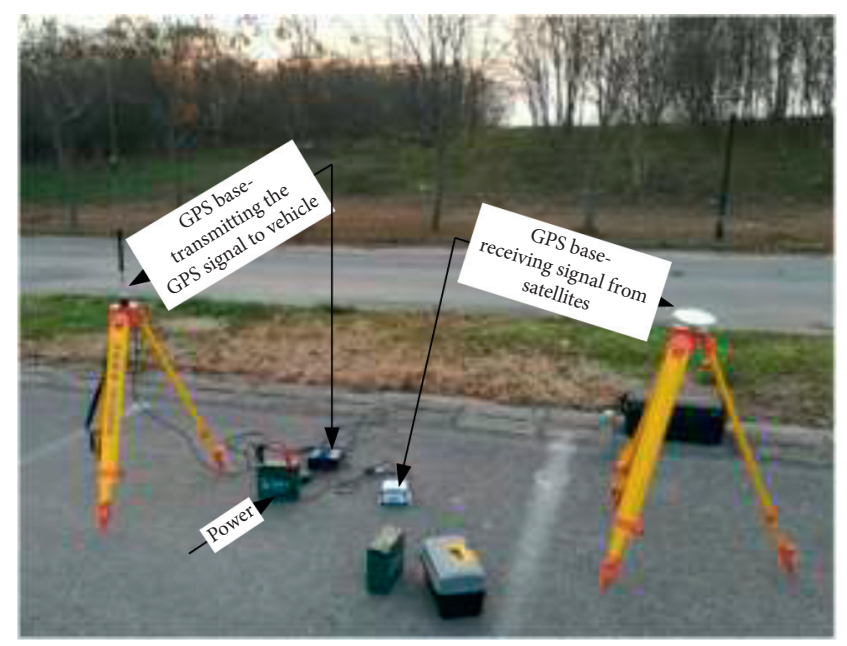

Figure 3: The GPS/IMU configuration.

maneuver corresponding to the driver yaw rate input. Note that steering wheel angle sensor (SAS) is employed to measure steering angle; then we can obtain the steering wheel angle $\delta_{h}$ in the field test. In addition, there is no steering motor on the vehicle. In the following test, the driver will release the steering wheel to simulate the situation when the steering motor fault happens. 


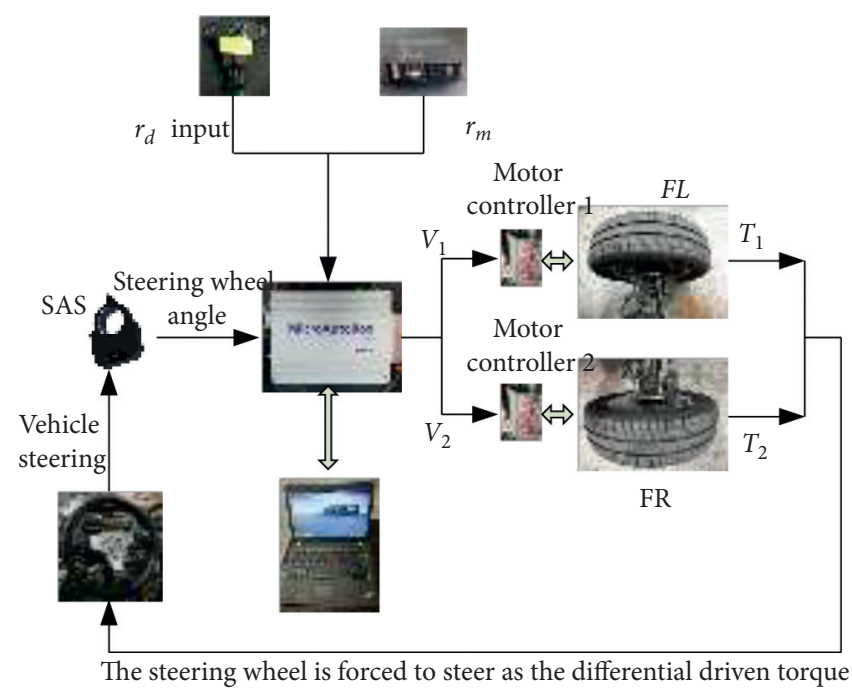

FIgURE 4: The flow chart of the proposed control schema.

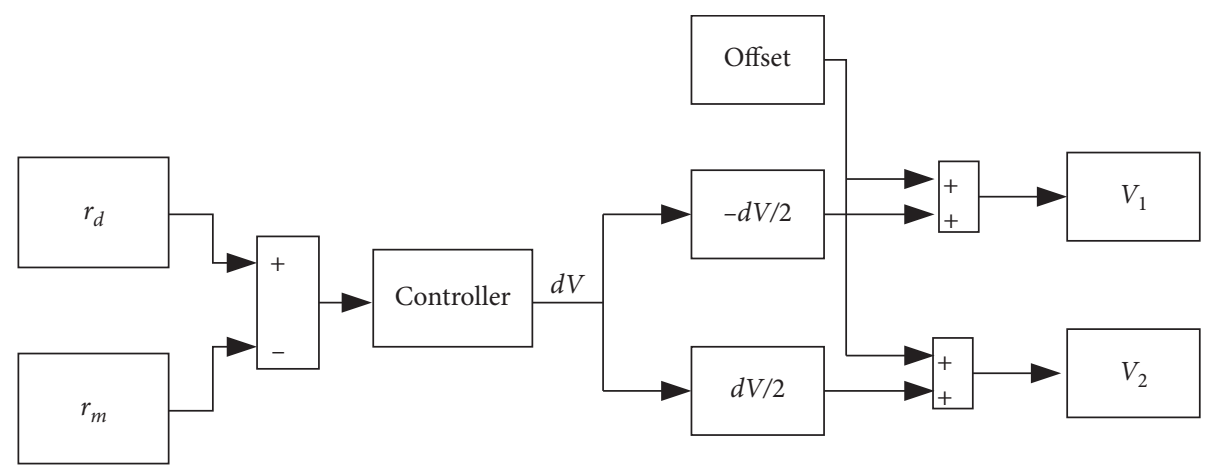

FIgURE 5: The PID control of the differential steering.

\section{Experimental Study of Differential Steering}

In this section, field test of the vehicle is presented to validate the effectiveness of the differential steering. In the tests, as seen in Figure 6, the vehicle runs well initially, and then the driver releases the steering wheel. Meanwhile, the copilot activates the differential steering control schema and gives the desired yaw rate signals to the controller. Without steering torque from the driver, the steering wheels are only regulated by the differential steering controller. The controller makes the vehicle track its desired yaw rate with the proposed control schema. Three different driving maneuvers are performed in the experiment and the results are in Figures 7-9, respectively. Note that, in these tests, the vehicle is only driven by the front-two in-wheel motors, and the rear-two in-wheel motors are shut off. The positive yaw rate indicates left steering directions, and vice versa.

4.1. Fixed Yaw Rate Tracking. In this test, the vehicle is supposed to track a fixed yaw rate $r_{d}=-7 \mathrm{deg} / \mathrm{s}$. In Figure $7(\mathrm{a})$, it is clear that, at the first few seconds, the actual yaw rate is zero, indicting that the vehicle is running in a straight line. Then the driver releases the steering wheel, and the copilot activates the differential steering and inputs the

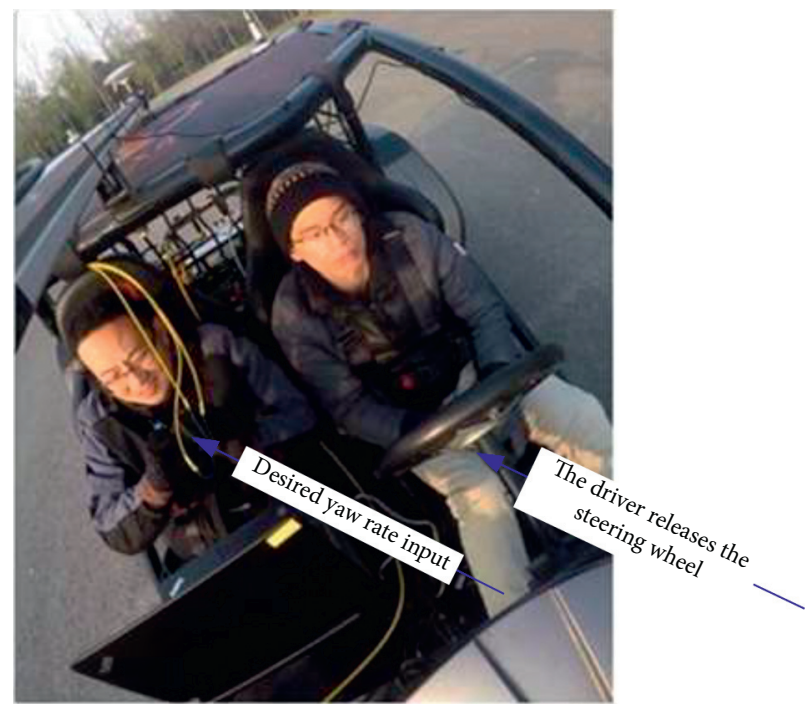

Figure 6: The field test of the differential steering.

desired yaw rate $r_{d}=-7 \mathrm{deg} / \mathrm{s}$. As seen in Figure 7(b), when the differential steering is activated, the control system quickly generates the control signals. Then the control voltage of the two in-wheel motors is separated to the 


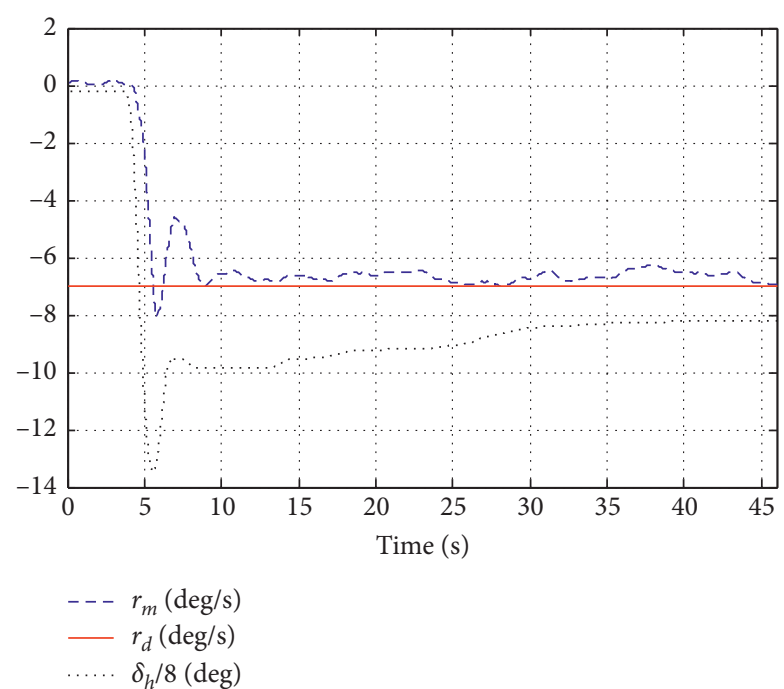

(a)

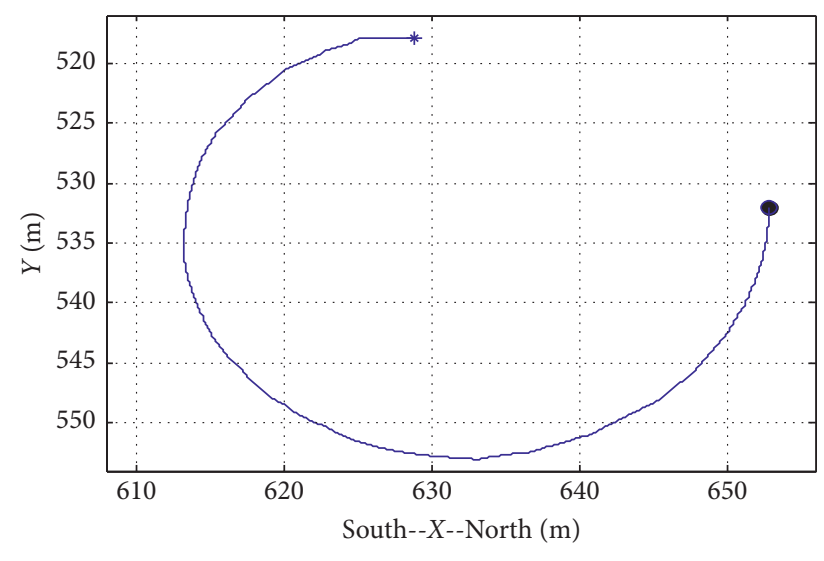

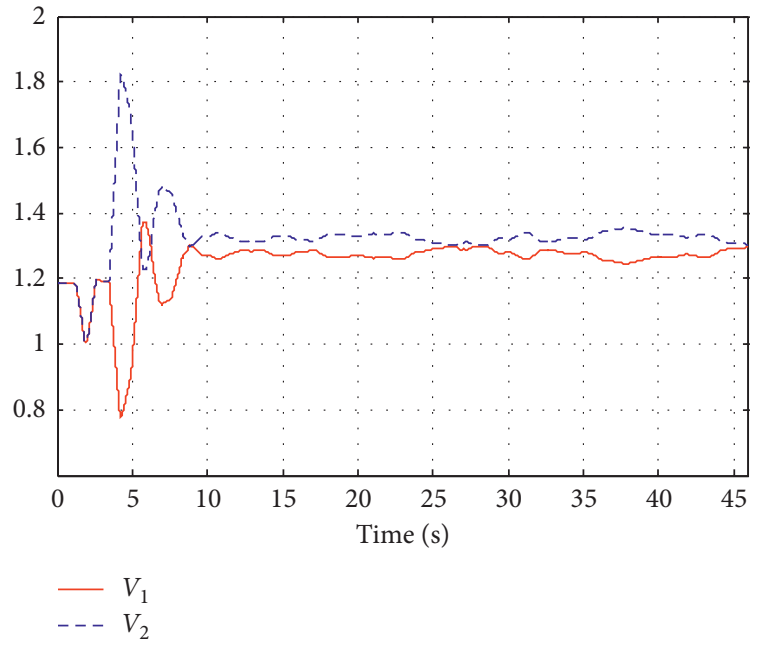

(b)

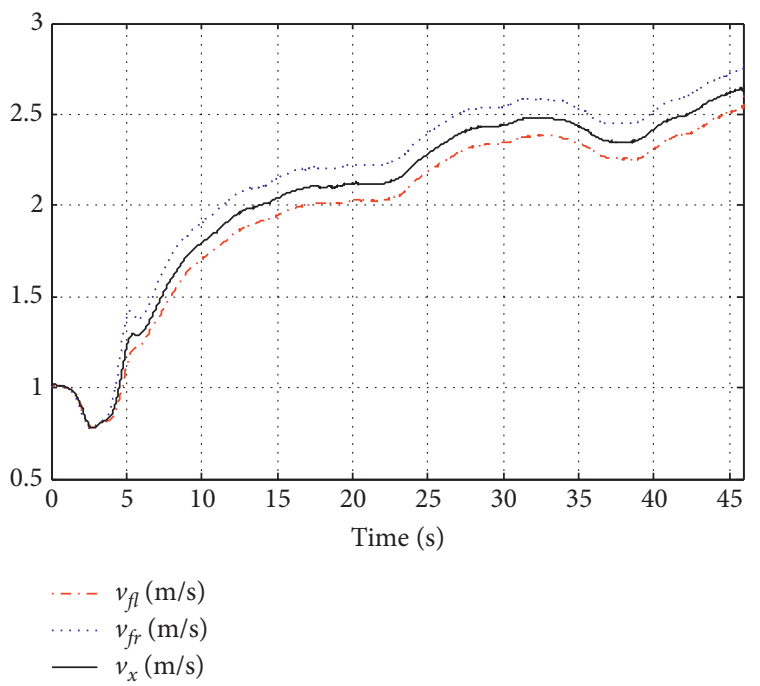

(d)

Figure 7: The result of the fixed yaw rate tracking. (a) Time (s). (b) Time (s). (c) South-X-North. (d) Time (s).

opposite direction. The control signal of front-right in-wheel motor $V_{2}$ increases and the front-left motor signal $V_{1}$ decreases. With the different control signals, the in-wheel motors generate the different torques, and thus the wheel speed is quite different as seen in Figure $7(\mathrm{~d})$. With such signals, the right motor generates larger speed than the left one, making the vehicle turn left and the actual yaw rate $r_{m}$ rapidly reaches $-7 \mathrm{deg} / \mathrm{s}$. Note that, in Figure $7(\mathrm{~d})$, the black solid line indicates the vehicle speed $\left(v_{x}\right)$. In Figure $7(\mathrm{c})$, the vehicle is approximately running in a straight line at the beginning ( $*$ means starting point; $\bullet$ means ending point) and then in a circle as $r_{m} \approx-7 \mathrm{deg} / \mathrm{s}$.

In Figure $7(a)$, it is clear that the overall yaw rate tracking performance is well. Because the driver's steering angle is relatively large compared to the yaw rate signal, we give the scaled steering wheel angle, that is, $\delta_{h} / 8$, as the black-dotted line in Figure 7(a). Without the driver intervention, the differential steering can still rotate steering wheel, achieving the similar effect of the electric power steering.

4.2. Yaw Rate Tracking with Lane-Change-like Maneuver. In this test, the vehicle is supposed to track a varied yaw rate with the lane-change-like maneuver. The results are given in Figure 8. As seen in zone A of Figure 8(a), the actual yaw rate is zero at the beginning. At $2 \mathrm{~s}$, the driver releases the steering wheel, and the copilot activates the differential steering and changes the desired yaw rate. When the differential steering is activated, the control system quickly generates the control signals, as in zone A of Figure 8(b). Then the control voltage of the two in-wheel motors is separated to the opposite direction. The control voltage signal of the front-right inwheel motor $V_{2}$ is larger than $V_{1}$ in zone $A$, indicating the left turning of the vehicle, as shown in zone $A$ of Figure $8(b)$. As the vehicle turns left, the right wheel has larger speed 


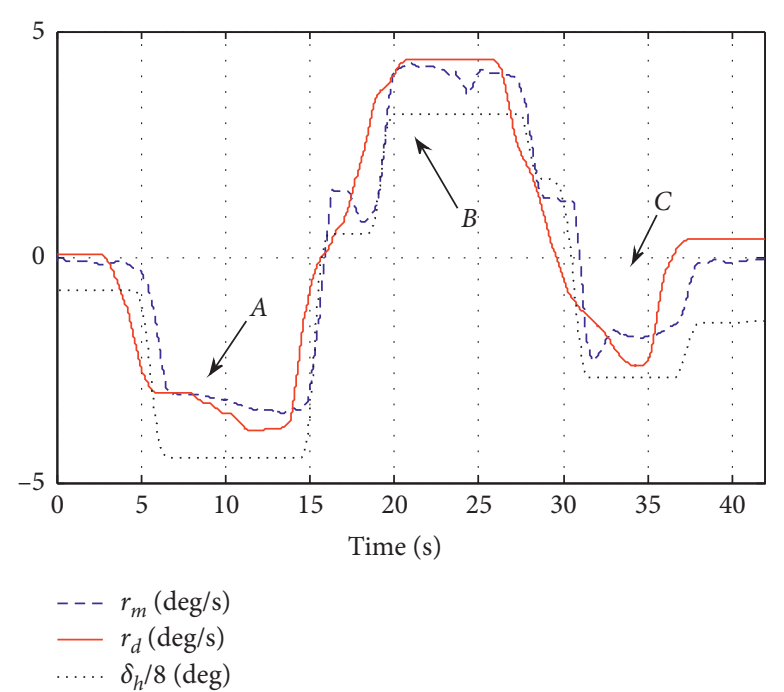

(a)

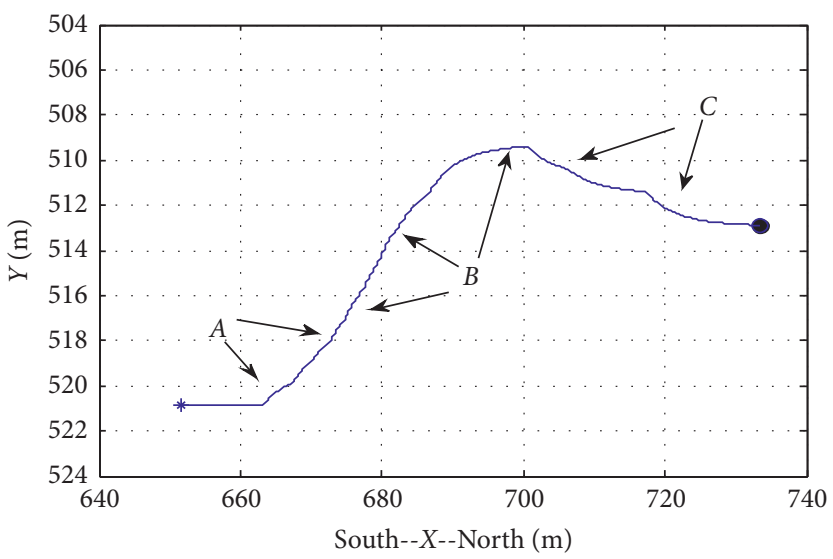

(c)

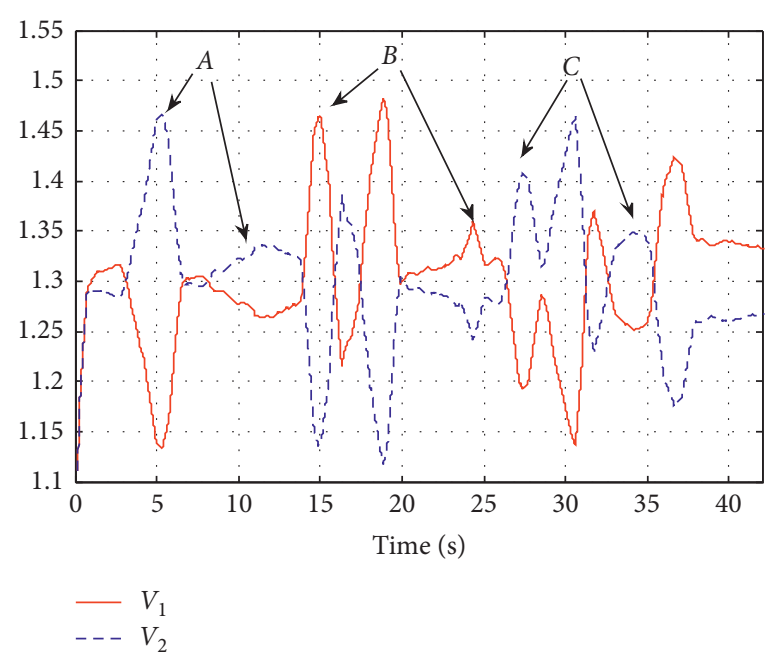

(b)

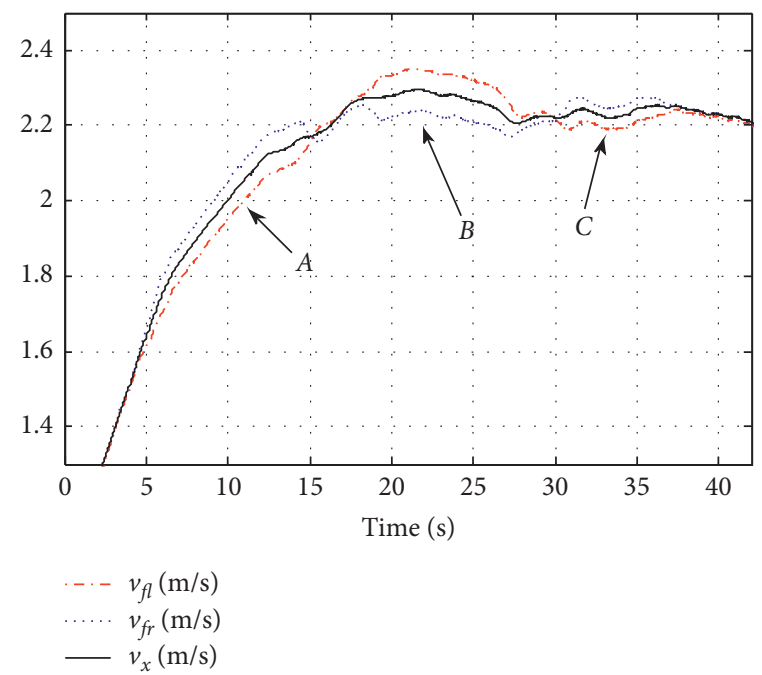

(d)

Figure 8: Yaw rate tracking with lane-change-like maneuver. (a) Time (s). (b) Time (s). (c) South-X-North. (d) Time (s).

$\left(v_{f r}\right)$ than the left $\left(v_{f l}\right)$ as in zone $A$ of Figure 8(d). The actual yaw rate tracks the desired value well in zone $A$. In zone $B$ of Figure 8(a), the driver changes the direction of the yaw rate, trying to amend the heading angle and let the vehicle make an opposite turn. Then the control system increases $V_{1}$ and decreases $V_{2}$ as in zone $B$ of Figure $8(\mathrm{~b})$, generating larger torque in the left wheel than in the right, and thereby the vehicle turns right as in zone $B$ of Figure $8(\mathrm{c})$. As the vehicle turns right, the left wheel has larger speed $\left(v_{f l}\right)$ than the right $\left(v_{f r}\right)$ as in zone B of Figure 8(d). The actual yaw rate tracks the desired value well in zone $B$.

In zone $C$ of Figure $8(a)$, the driver changes the yaw rate direction again to amend the heading angle and let the vehicle move in straight line. Then the system increases $V_{2}$ and decreases $V_{1}$ as in zone $C$ of Figure 8(b), causing larger torque in the right wheel than in the left and thereby stopping the vehicle's right turning trend as in zone $C$ of Figure 8(c). In zone $C$ of Figure 8, the right wheel has larger speed $\left(v_{f r}\right)$ than the left $\left(v_{f l}\right)$. Even though the operations in zone $C$ are not perfect, the actual yaw rate tracks the desired value well in zone $C$ and the lane-change maneuver is still acceptable.

4.3. Yaw Rate Tracking with J-Turn-like Maneuver. In this test, the vehicle is supposed to track a varied yaw rate with the J-turn-like maneuver. In zone $A$ of Figure 9(a), the actual yaw rate is zero at the first few seconds. At $5 \mathrm{~s}$, the driver releases the steering wheel, and the copilot activates the differential steering and inputs the desired yaw rate. In zone $A$, the desired yaw rate becomes $-3 \mathrm{deg} / \mathrm{s}$. Then the system redistributes the control voltage of the front-two in-wheel motors, as in Figure 9(b). The control signal of the frontright in-wheel motor $V_{2}$ is larger than $V_{1}$ in zone $A$, indicating the left turning of the vehicle, as in Figure 9(c). As the vehicle turns left, the right wheel has larger speed $\left(v_{f r}\right)$ than 


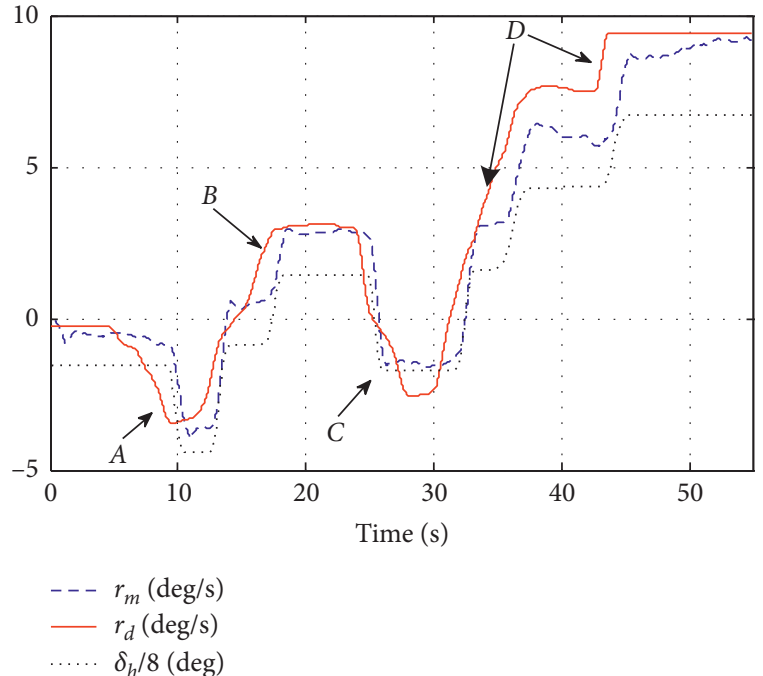

(a)

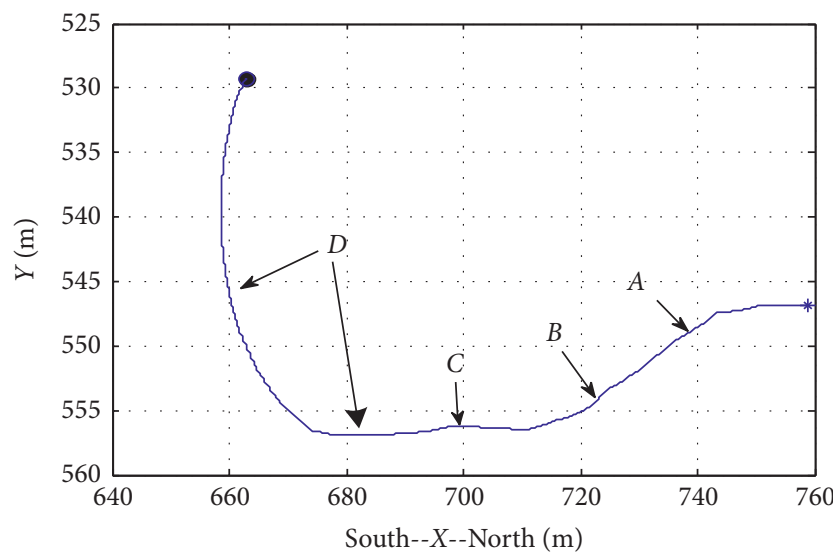

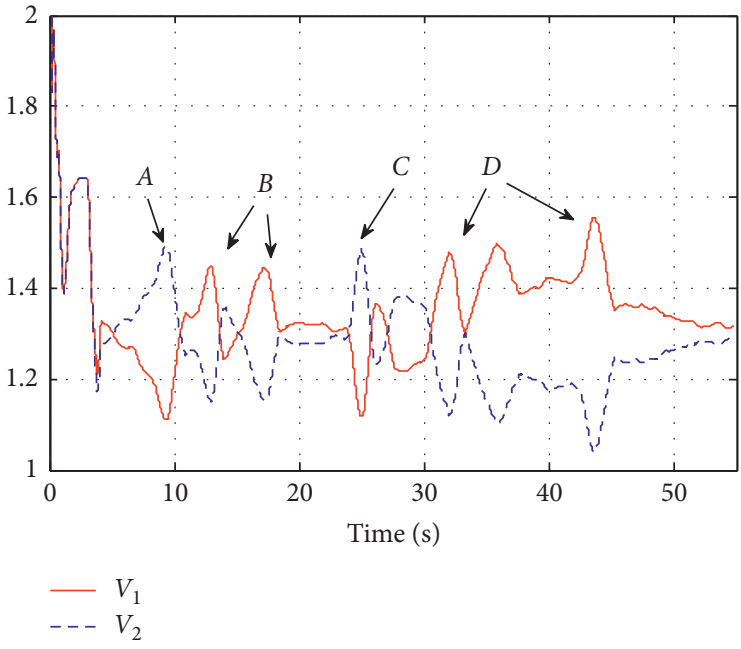

(b)

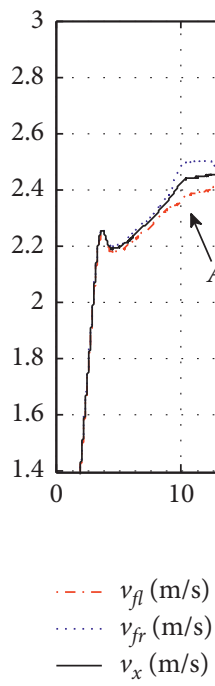

(d)

Figure 9: Yaw rate tracking with J-turn-like maneuver. (a) Time (s). (b) Time (s). (c) South-X-North. (d) Time (s).

the left $\left(v_{f l}\right)$ as in Figure 9(d). The actual yaw rate tracks the desired value well in zone A.

In zone B of Figure 9(a), the driver tries to change the yaw rate direction to amend the heading angle and let the vehicle make an opposite turn. Then the system increases $V_{1}$ and decreases $V_{2}$ as in zone $B$ of Figure 9(b), causing larger torque in the left wheel than in the right, and thereby the vehicle turns right as in zone $B$ of Figure 9(c). As the vehicle slightly turns right, the left wheel has larger speed $\left(v_{f l}\right)$ than the right $\left(v_{f r}\right)$ as in zone $B$ of Figure $9(\mathrm{~d})$. The actual yaw rate tracks the desired value well in zone $B$.

In zone $C$ of Figure $9(\mathrm{a})$, the driver tries to amend the heading angle and let the vehicle turn left. Then the system increases $V_{2}$ and decreases $V_{1}$ as in zone C of Figure 9(b), causing larger torque in the right wheel than in the left and thereby stopping the vehicle's right turning trend as in zone
$C$ of Figure $9(\mathrm{c})$. As the vehicle stops turning right, the right wheel has larger speed $\left(v_{f r}\right)$ than the left $\left(v_{f l}\right)$ as in zone $C$ of Figure $9(\mathrm{~d})$.

In zone $D$ of Figure $9(\mathrm{a})$, the driver tries a J-turn maneuver in right turning. Then the system increases $V_{1}$ and decreases $V_{2}$ as in zone $D$ of Figure 9(b), causing larger torque in the left wheel than in the right, and thereby the vehicle turns right as in zone $D$ of Figure $9(\mathrm{c})$. As the vehicle turns right sharply, the left wheel has larger speed $\left(v_{f l}\right)$ than the right $\left(v_{f r}\right)$ as in zone $D$ of Figure $9(\mathrm{~d})$. With the higher speed and sharp steering in the J-turn test, the operations in zone $D$ are not perfect. However, under such circumstance, the performance of yaw rate tracking and the J-turn trajectory in this test is still acceptable. Therefore, the feasibility and effectiveness of the differential are proven by the field tests. 


\section{Conclusion}

As the driven torque of the FWID EV between the front-left and front-right wheels can be independently controlled, this paper designs the differential steering as the backup steering power when the SBW system fails its regular function. To test the real performance of the differential steering, a FWID EV is manufactured and the corresponding control schema is designed. The field test of the vehicle yaw rate tracking is performed under the cooperation of the driver and the copilot. The fixed yaw rate tracking and varied yaw rate tracking maneuvers are employed in the tests of the FWID EV. In the fixed yaw rate tracking, the vehicle can track the desired yaw rate well with differential steering. In addition, the vehicle can track the varied yaw rate with proposed differential steering. The test results verify the effectiveness of the proposed differential steering.

\section{Data Availability}

The data used to support the findings of this study are available from the corresponding author upon request.

\section{Conflicts of Interest}

The authors declare no conflicts of interest.

\section{Acknowledgments}

This work was partly supported by the Natural Science Foundation of Guangxi Province (2018GXNSFAA281271, 2020GXNSFAA297031, and Guike AD18281063).

\section{References}

[1] H. Zhao, B. Ren, H. Chen, and W. Deng, "Model predictive control allocation for stability improvement of four-wheel drive electric vehicles in critical driving condition," IET Control Theory \& Applications, vol. 9, no. 18, pp. 2688-2696, 2015.

[2] J. Hu, Y. Yang, M. Jia, Y. Guan, C. Fu, and S. Liao, "Research on harmonic torque reduction strategy for integrated electric drive system in pure electric vehicle," Electronics, vol. 9, no. 8, p. $1241,2020$.

[3] H. Jing, R. Wang, J. Wang, and N. Chen, "Robust $H_{\infty}$ dynamic output-feedback control for four-wheel independently actuated electric ground vehicles through integrated AFS/ DYC," Journal of the Franklin Institute, vol. 355, no. 18, pp. 9321-9350, 2018.

[4] L. Zhao, S. Lu, and B. Zhang, "Game-based hierarchical cooperative control for electric vehicle lateral stability via active four-wheel steering and direct yaw-moment control," Energies, vol. 12, no. 17, p. 3339, 2019.

[5] G. Zhang, H. Zhang, X. Huang, J. Wang, H. Yu, and R. Graaf, "Active fault-tolerant control for electric vehicles with independently driven rear in-wheel motors against certain actuator faults," IEEE Transactions on Control Systems Technology, vol. 24, no. 5, pp. 1557-1572, 2016.

[6] W. Zhao, X. Qin, and C. Wang, "Yaw and lateral stability control for four-wheel steer-by-wire system," IEEE/ASME Transactions on Mechatronics, vol. 23, no. 6, pp. 2628-2637, 2018.
[7] H. Wang, Z. Man, H. Kong et al., "Design and implementation of adaptive terminal sliding-mode control on a steer-by-wire equipped road vehicle," IEEE Transactions on Industrial Electronics, vol. 63, no. 9, pp. 5774-5785, 2016.

[8] N. Wada, K. Fujii, and M. Saeki, "Reconfigurable fault-tolerant controller synthesis for a steer-by-wire vehicle using independently driven wheels," Vehicle System Dynamics, vol. 51, no. 9, pp. 1438-1465, 2013.

[9] R. Wang, H. Jing, C. Hu, M. Chadli, and F. Yan, "Robust $H_{\infty}$ output-feedback yaw control for in-wheel motor driven electric vehicles with differential steering," Neurocomputing, vol. 173, pp. 676-684, 2016.

[10] C. Hu, R. Wang, F. Yan, and H. Karimi, "Robust composite nonlinear feedback path following control for independently actuated autonomous vehicles with differential steering," IEEE Transactions on Transportation Electrification, vol. 2, no. 3, pp. 312-321, 2016.

[11] M. Kuslits and D. Bestle, "Modelling and control of a new differential steering concept," Vehicle System Dynamics, vol. 57, no. 4, pp. 520-542, 2019.

[12] J. Wang, Q. Wang, L. Jin, and C. Song, "Independent wheel torque control of $4 \mathrm{WD}$ electric vehicle for differential drive assisted steering," Mechatronics, vol. 21, no. 1, pp. 63-76, 2011.

[13] J. Wang, T. Yan, Y. Bai, Z. Luo, X. Li, and B. Yang, "Assistance quality analysis and robust control of electric vehicle with differential drive assisted steering system," IEEE Access, vol. 8, pp. 136327-136339, 2020.

[14] W. Zhao and H. Zhang, "Coupling control strategy of force and displacement for electric differential power steering system of electric vehicle with motorized wheels," IEEE Transactions on Vehicular Technology, vol. 67, no. 9, pp. 8118-8128, 2018.

[15] J. Ni and J. Hu, "Dynamic modelling and experimental validation of a skid-steered vehicle in the pivotal steering condition," Proceedings of the Institution of Mechanical Engineers Part D-Journal of Automobile Engineering, vol. 231, no. 2, pp. 225-240, 2017.

[16] N. Gupta, C. Ordonez, and E. Collins, "Dynamically feasible, energy efficient motion planning for skid-steered vehicle," Autonomous Robots, vol. 41, no. 2, pp. 453-471, 2017.

[17] P. Oke and S. Nguang, "Robust $H_{\infty}$ Takagi-Sugeno fuzzy output-feedback control for differential speed steering vehicles," Proceedings of the Institution of Mechanical Engineers Part D-Journal of Automobile Engineering, vol. 234, no. 12, pp. 2822-2835, 2020.

[18] T. Xu, X. Ji, Y. Liu, and Y. Liu, "Differential drive based yaw stabilization using MPC for distributed-drive articulated heavy vehicle," IEEE Access, vol. 8, pp. 104052-104062, 2020.

[19] S. Wang, G. Shi, and Y. Lin, "Integrated hierarchical control strategy of active suspension and differential assisted steering system for electric-wheel vehicle," International Journal of Vehicle Design, vol. 81, no. 3-4, pp. 212-240, 2019.

[20] L. Xiong, S. Huang, Y. Chen, G. Yang, and R. Zhang, "A research on motion tracking control for unmanned ground vehicle with wheeled skid steering," Automotive Engineering, vol. 37, no. 10, pp. 1109-1116, 2015.

[21] C. Ahn, H. Peng, and H. Tseng, "Robust estimation of road frictional coefficient," IEEE Transactions on Control Systems Technology, vol. 21, no. 1, pp. 1-13, 2013. 